\title{
Noise-induced Cochlear Synaptopathy in Dental Prosthesis Students
}

\author{
(D) Bünyamin Çıldır1, Suna Tokgöz Yılmaz² \\ 1Ankara Yıldırım Beyazıt University Health Sciences Faculty, Department of Language and Speech Therapy, Ankara, Turkey \\ 2Ankara University Health Sciences Faculty, Audiology Department, Ankara, Turkey
}

\section{Abstract}

BACKGROUND/AIMS: Noise causes damage to cochlear hair cells and loss of sensitivity at low volume sounds. Hidden hearing loss is a functional disorder that can be seen in individuals with noise exposure history but no permanent threshold loss. We aimed to determine which tests can be used to diagnose hidden hearing loss in the dental prosthesis associate degree students with normal hearing who are exposed to noise.

MATERIAL AND METHODS: Ninety individuals between the ages of 19-35 whose pure tone average is within normal limits were included in our study. These individuals were divided into two groups according to the noise exposure score as high-risk group ( $\mathrm{n}=45)$ and low-risk group $(n=45)$. Auditory brainstem response (ABR) and amplitude modulation detection tests were performed with and without background noise after standard audiometric tests and otoacoustic emission suppression measurement.

RESULTS: The otoacoustic emission suppression values of individuals with high-risk group were found significantly lower than those with low-risk group. As the stimulus level increased, the differentiations (amplitude increase and latency decrease) in the first wave of ABR without background noise were observed in individuals with the low-risk group. The recognition threshold score which was modulated to the amplitude was found to be lower in the presence of background noise than in the absence of background noise for all participants.

CONCLUSION: Although noise exposure does not result in any permanent differences in hearing thresholds, the otoacoustic emission suppression values, the differentiations of the first wave and the amplitude modulation detection values can provide useful information in the diagnosis of hidden hearing loss in individuals with normal hearing.

Keywords: Hidden hearing loss, auditory brainstem response, otoacoustic emission suppression, amplitude modulation detection

\section{INTRODUCTION}

Exposure to high intensity noise may cause unwanted sounds to be masked in speech and communication, as well as long-term exposure to such noises can cause physical (such as temporary hearing loss, increased blood pressure) and mental effects (such as stress, anxiety, mental difficulty) in humans. ${ }^{1-3}$ These effects may vary depending on the duration of exposure to noise, the distance to the source and the sensitivity of the person. ${ }^{1,3}$

The loss of the synaptic connection between the inner hair cell and spiral ganglion cells after noise exposure is the first pathological finding of temporary hearing loss. ${ }^{4}$ This deterioration is called "Synaptopathy" due to the connection loss between lesion area

To cite this article: Çıldır B, Tokgöz Yılmaz S. Noise-Induced Cochlear Synaptopathy in Dental Prosthesis Students.

Cyprus J Med Sci 2021

ORCID iDs of the authors: B.Ç. 0000-0002-5632-1650; S.T.Y. 0000-0002-4656-099X

Address for Correspondence: Bünyamin ÇILDIR

E-mail: bunyamin.cildir@gmail.com

ORCID ID: orcid.org/0000-0002-5632-1650
Received: 18.02 .2021

Accepted: 13.06.2021 
inner hair cell ribbon synapses and afferent hearing nerves or is called as "hidden hearing loss" since it does not affect hearing thresholds. ${ }^{4}$

Hidden hearing loss is a functional disorder that can be seen in individuals with noise exposure history but no permanent threshold loss. ${ }^{5}$ We can learn about functional distortions in outer hair cells, especially, by determining the lowest discernible hearing threshold levels in a given frequency region with the use of pure tone audiometry. ${ }^{6}$ Hidden hearing loss is assumed to affect low-spontaneous rate fibers with high thresholds, which are responsible for encoding moderate to high noise intensity. In this pathology which occurs after noise exposure, due to the peripheral loss of hearing nerve fibers with a high threshold and low spontaneous rate, no deterioration occurs in low-intensity sounds. ${ }^{7}$ High-intensity sounds, consistent with low SR fiber loss, can be associated with non-abnormal results. ${ }^{8}$ It has been indicated that hidden hearing loss cannot be determined by audiometric results but there is a decrease in the amplitude of wave I of the auditory brainstem responses to moderate-to-high intensity stimuli ${ }^{8}$, and amplitude modulation detection values. ${ }^{9}$ Liberman et $\mathrm{al}^{10}$, normal hearing individuals with low-risk of noise exposure and normal hearing individuals with high-risk were compared in terms of cochlear synaptopathy, and the amplitude of wave I of the auditory brainstem responses was not found to be decreased in the high risk group. In another study conducted on individuals with and without tinnitus, it was reported that the amplitudes of individuals with tinnitus decreased more with wave I of the auditory brainstem responses compared to the group without tinnitus. ${ }^{11}$ Dentists or dental assistants might be exposed to different noise levels and types while working in dental offices or laboratories. ${ }^{12}$

In our study, we aimed to determine whether auditory brainstem responses and distortion suppression of otoacoustic emissions and amplitude modulation detection tests and speech in noise test would be useful in order to determine whether there is latent hearing loss in individuals with dental prosthesis associate degree students with normal hearing. At the same time, the results of dental prosthesis associate degree students (dental auxiliaries) were compared with the results of individuals with normal hearing who were not exposed to noise, and the damage caused by noise exposure to the human hearing system was evaluated.

\section{MATERIAL AND METHODS}

Our study was approved by the $x x x x x x x x x x x x x x x x x x x x x x$ Ethics Committee and implemented according to the Helsinki Declaration (Approve no:56/15). The individuals who applied for the occupational health surveillance were informed told about the study about the study and their consent was obtained from those who agreed to participate.
Participants: A total of 90 individuals, who are aged between $19-35$ years (23.45 \pm 3.67$)$, with dental prosthesis associate degree students with high-risk ( $n=45$; noise exposure score: 5 and above) exposed to noise between the ages of 19-35 and with low-risk normal hearing with a low noise exposure $(n=45$; noise exposure score: 4 and above) according to one minute noise questionnaire score. Our study was conducted with xxxxxxx University, Vocational High School, dental prosthesis associate degree students and undergraduate students with normal hearing and no history of noise exposure. The study was conducted in

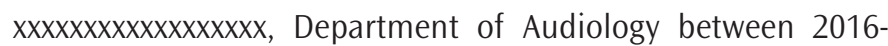
2018 years. Individuals who are over 18 years old and without hearing loss history, neurological disorders, and tinnitus were included in the study (hearing thresholds $\leq 20 \mathrm{~dB}$ HL at 125-12000 $\mathrm{Hz}$ ). Four participants with hearing thresholds higher than $20 \mathrm{~dB}$ HL were excluded from the study. After otoscopy, all participants were tested by pure tone audiometry, immitansmetry, speech in noise test, DPOAE suppression measurement, auditory brain stem response, and amplitude modulation detection tests with and without background noise. The measurements of all participants were made when the schools entered the semester break. None of the participants entered the laboratory within this time (approximately 3 weeks).

Evaluation of Noise Exposure and Sensitivity: Participants' noise exposure score were determined by 1-minute noise screen questionnaire. ${ }^{13}$ Those with a score of 5 and above were evaluated as high-risk individuals and those with a score of 4 or below were evaluated as low-risk individuals according to questionnaire. Besides, to evaluate the participants' noise sensitivity we used Turkish validity and reliability of Weinstein noise sensitivity, the total score is calculated by giving 1 to 6 points (agree/disagree) to 21 questions in this scale (highest score 126). ${ }^{14}$ At the same time, the noise level of the dental prosthesis laboratory was measured using the Larson Davis system 824 sound level meter. Noise level of laboratory were measured at every 10 minutes (10:00-12:00 and 13:00-14:00) during about three month (without weekends) from the same point in the middle of the laboratory while all devices in the laboratory were working. Measurements were made a total of 18 times every day.

\section{Speech in Noise Test}

This test is carried out using monosyllabic word lists in background noise such as narrowband and white noise. ${ }^{15,16}$ This test can be performed at different signal to noise ratios as well as at fixed signal to noise ratios. ${ }^{17}$ In our study, 50-word monosyllabic word lists were made at $40 \mathrm{~dB}$ SL, with a constant $+10 \mathrm{~dB}$ signal/noise ratio to the ear to be tested for both noise and speech stimuli. Each ear was evaluated separately.

Pure Tone Audiometry and Immitancemeter Measurements: Pure tone audiometry were performed with Sennheiser TDH 49 $P$ supraural headset in the range of 0.125 to $8 \mathrm{kHz}$ and with 
Sennheiser HDA 200 circumaural headphones in the range of 8 to $12 \mathrm{kHz}$ according to the British Society of Audiology (2011). Participants' middle ear pressure values were between \pm 50 daPa and performed by GSI TympStar device.

\section{Contralateral Suppression of Distortion Product Otoacoustic} Emissions: Distortion Product Otoacoustic Emission (DPOAE) is an electro-acoustic measurement that reflects the mechanical properties of the outer hair cells (OHC). DPOAE test is performed by simultaneously transmitting two pure tone sounds (to the ear at different frequencies $\mathrm{f} 1$ and $\mathrm{f} 2$ using an $\mathrm{f} 2 / \mathrm{f} 1$ ratio of 1.2$).{ }^{18}$ The DPOAE amplitude was chosen when L1 (75 dB SPL) was $10 \mathrm{~dB}$ higher than L2 (65 dB SPL). The contralateral DPOAE suppression test causes suppression of $\mathrm{OHCs}$ with contralateral noise. The contralateral DPOAE suppression test can either be performed by decreasing the amplitude (Input-Output-I/O function) or by changing the frequency values of the constant amplitude (DP-Gram). ${ }^{19}$ We used Otometrics (Denmark, Taastrup) Capella Distortion Product Otoacoustic Emission. DP-gram was obtained at octave frequencies of 200, 2383, 3359, $4004 \mathrm{~Hz}$ at $60 \mathrm{~dB}$ SPL broadband-contralateral white noise $\mathrm{Hz}$ using contralateral TDH39 headphone.

Auditory Brainstem Response: Auditory brainstem (ABR) measurements of the participants were performed in a natural sleep state in the Faraday cage test room using Vivasonic (Canada, Toronto) Integrety ${ }^{\text {TM }}$ V500 Auditory Diagnostic System in two channels. The active electrode $\left(\mathrm{F}_{\mathrm{Z}}\right)$ was placed on the upper part of the forehead, the ground electrode $\left(\mathrm{F}_{\mathrm{pz}}\right)$ was placed on the lower part of the forehead, one of the reference electrodes was placed on the left (M1) and the other was placed on the right mastoid (M2) region (electrode skin impedance below 3 $k \Omega$ ). The click stimuli (with 1024 repeats) at alternative polarity were transmitted through ER-3A insert headphones, using a 30 $\mathrm{Hz}$ high-pass filter (highpass). ABR recordings were kept by using a $100 \mu$ s stimulus at a rate of $9.1 \mathrm{~Hz}$ (rate) at 70, 80, 90, $99 \mathrm{~dB}$ $\mathrm{nHL}$ levels to the opposite ear, for both cases of a $55 \mathrm{~dB} \mathrm{nHL}$ broadband mask and without a mask.

\section{Amplitude Modulation Detection Test: Amplitude} modulation detection test (AMDT) is used to detect amplitude modulation sounds that are assumed to decrease after noise exposure because of impairment in individuals' over-threshold responses. AMDT was performed with and without contralateral narrowband noise $\left(40 \mathrm{~dB}\right.$ SPL). ${ }^{20}$ The bandwidth of narrowband noise was set to $1 / 3$ of an octave in this study. A sinusoidal sound was modulated to $19 \mathrm{~Hz}$ with a carrier frequency of $5 \mathrm{kHz}$ at $75 \mathrm{~dB}$ SPL level was used. The AMDT test was performed using the Parameter Estimation by Sequential Testing (3 interval-3 alternative selection methods) and the p-value was taken into consideration as 0.75 . One of three randomly transmitted sounds was modulated to stimulus amplitude and the other two were not modulated. While the target tone was being transmitted, the AM depth was initially set as $6 \mathrm{~dB}(50 \%)$ and was adaptively randomized until the final modulation change size reached at $0.45 \mathrm{~dB}$; the mean value of the last two steps was accepted as the threshold. ${ }^{21}$ We was used AMDT software with the Matlab 2019.b program.

Statistical Analysis: Data analysis was performed using SPSS v.23.0 (Statistical Package for Social Sciences) (SPSS Inc., Chicago, IL, US). The normal distribution of our study was examined with the Shapiro-Wilk test. A chi-square test was used to compare demographic findings. The non-parametric Mann-Whitney $U$ test was used for the comparison between the high-risk and lowrisk groups. Wilcoxon test was used in-group comparison and Spearman test was used for the relation between two variables. The $p$-value for statistical significance was accepted as $<0.05$.

\section{RESULTS}

Noise Exposure and Sensitivity: The average noise level of the dental laboratory was $97 \pm 11.3 \mathrm{~dB}$ (A) for 10:00 o'clock and 102 \pm 9.3 $d B(A)$ for 14:00 o'clock. These hours are when the laboratory is very busy. Of the 45 participants in the high-risk group (dental prosthesis associate degree students), 41 reported that they were exposed to sounds such as wedding halls, ambulances, and traffic noise during the day. $85 \%$ of the individuals in the low-risk group stated that they were exposed to traffic noise. As shown in Figure 1, 1-minute noise screen scores of individuals in the highrisk group $(7.29 \pm 1.23)$ were found to be statistically higher than the low-risk group 's scores $(2.81 \pm 1.35)(p=0.001)$. Weinstein's noise sensitivity scale scores of the individuals in the high-risk group (65.44 \pm 7.53$)$ were statistically higher than the scores of the low-risk group (34.16 \pm 8.14$)(p=0.011)$ (Figure 2). A positive and high correlation was observed between the 1-minute noise screen and Weinstein's noise sensitivity scale of all individuals $(r=0.822, p=0.001)$.

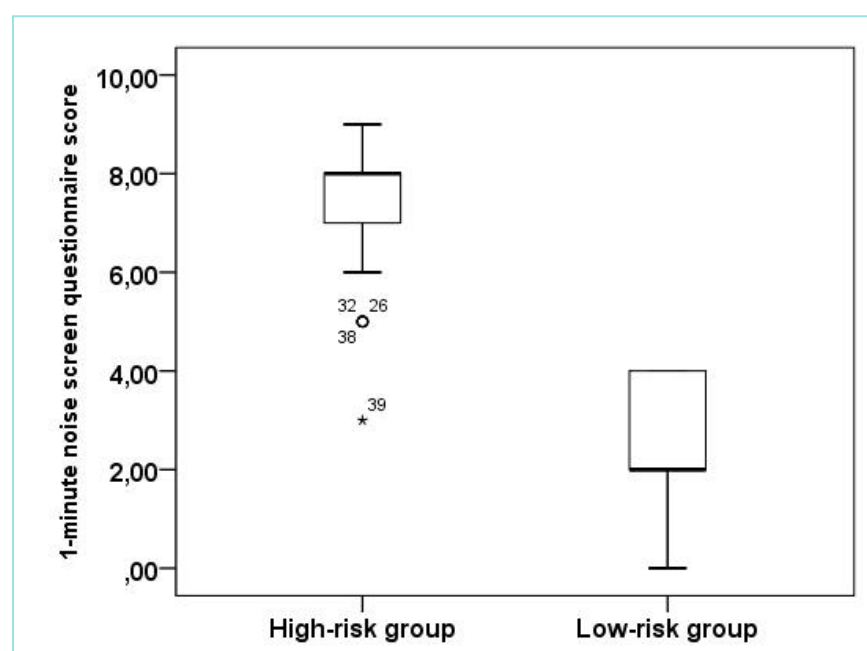

Figure 1. 1-minute noise screen questionnaire findings of the both groups, Error bars show the standard deviation. 
Pure Tone Audiometry: All participants have normal hearing thresholds (equal to or less than $20 \mathrm{~dB}$ HL at 125-8000 Hz octave frequencies. No statistical difference was observed between the hearing thresholds of the both groups ( $p>0.05$ ).

\section{Speech in Noise Test:}

Although no statistically significant difference was found between the scores of speech understanding in noise of the high and low-risk groups for both ears, the scores of the high-risk group were lower than the scores of the low-risk group.

\section{Contralateral Suppression of Distortion Product Otoacoustic}

Emissions: Contralateral DPOAE suppression amounts were calculated at all frequencies and were found $0.84 \mathrm{~dB}$ SPL for individuals with high-risk, $2.02 \mathrm{~dB}$ SPL for individuals with lowrisk. The difference in the contralateral DPOAE suppression amounts between the two groups was statistically significant $(p=0.014)$.

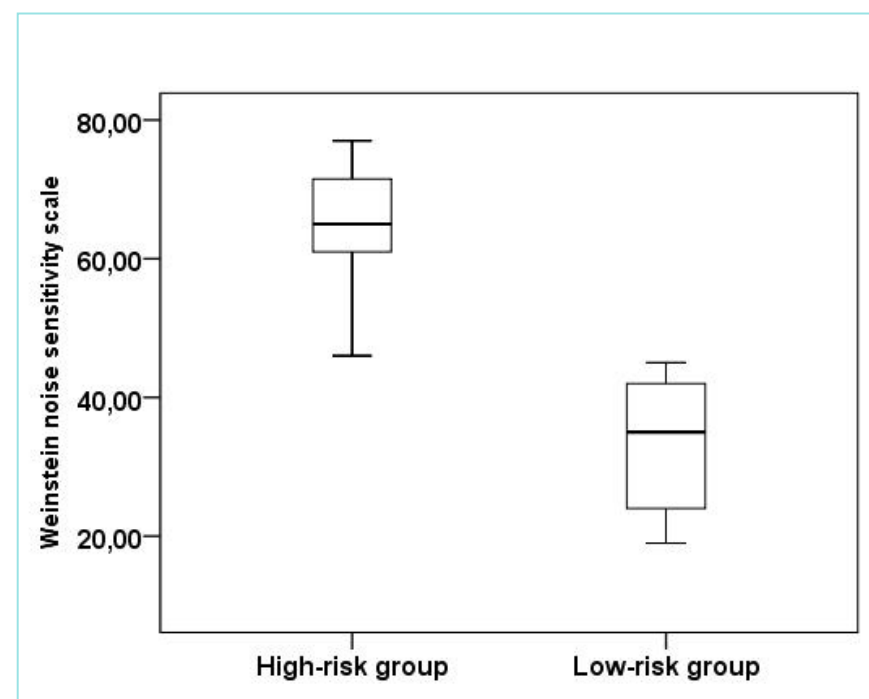

Figure 2. Weinstein noise sensitivity scale findings of the both groups, Error bars show the standard deviation.
Auditory Brainstem Responses: ABR records of all participants were made on the right ear of 68 patients and the left ear of 16 patients. While the amplitude of wave I of ABR with mask was statistically smaller than the amplitude value obtained without mask at $99 \mathrm{~dB} \mathrm{nHL}$ in the high-risk group ( $\mathrm{z}=-3.087, \mathrm{p}=0.002$ ), the amplitude was found to be bigger at the $80 \mathrm{~dB} \mathrm{nHL}$ without mask ( $z=-3.155, p=0.002$ ) (Table 1). A negative value was found in the amplitude of wave I of ABR in 10 individuals in the lowrisk group and 8 individuals in the high-risk group. The latency of wave I was obtained with mask was statistically significantly shorter than the latency without mask at $90 \mathrm{~dB} \mathrm{nHL}(\mathrm{z}=-2.178$, $\mathrm{p}=0.029)$ and $99 \mathrm{~dB} \cap \mathrm{HL}(\mathrm{z}=-2.449, \mathrm{p}=0.014)$ in the high-risk group. There was no statistically significant prolongation of the ABR wave $V$ latency with mask in the high-risk group ( $p>0.05)$.

As shown in Table 2, for the low-risk group; the wave I amplitude ( $\mathrm{z}=-2.587, \mathrm{p}=0.010)$ at $70 \mathrm{~dB} \mathrm{nHL}$ and the wave III amplitude at $90 \mathrm{~dB} \mathrm{nHL}(\mathrm{z}=-2.807, \mathrm{p}=0.005)$ values were found to be statistically lower with mask. There was no statistical difference between the amplitude and latency values of the wave I, III and $\mathrm{V}$ with mask and without mask at other (80 and $99 \mathrm{~dB}$ $\mathrm{nHL}$ ) intensity levels $(p>0.05)$. Although there was no statistical difference, it was observed that the amplitude increased as the stimulus intensity level raised.

For the high-risk group; the wave $V$ amplitude was found to be smaller with mask than without mask at $99 \mathrm{~dB} \mathrm{nHL}(\mathrm{z}=-2.562$, $\mathrm{p}=0.010)$ and $90 \mathrm{~dB} \mathrm{nHL}(\mathrm{z}=-1.999, p=0.046)$, but it was found to be bigger at $80 \mathrm{~dB} \mathrm{nHL}(z=3.905, p=0.001)$ (Table 1).

There were statistically significant differences in the wave I amplitude at $70 \mathrm{~dB} n \mathrm{HL}(\mathrm{U}=288, \mathrm{p}=0.001), 80 \mathrm{~dB} \mathrm{nHL}(\mathrm{U}=586.5$, $p=0.011)$ and $99 \mathrm{~dB} \mathrm{nHL}(\mathrm{U}=635.5, p=0.035)$ with mask between two groups. However, there was no difference at all stimulus levels without mask (Tables 1 and 2$)(p>0.05)$.

Amplitude Modulation Detection Threshold (AMDT): For both groups, the AMDT scores were evaluated both with mask and without mask (Table 3). AMDT values with mask were

Table 1. Auditory brainstem responses amplitude with/without mask at different stimulus level for the high-risk group

\begin{tabular}{|c|c|c|c|c|c|c|c|}
\hline Mask & Stimulus level & $\begin{array}{l}\text { Wave I } \\
\text { Mean } \pm \text { SD }\end{array}$ & p & $\begin{array}{l}\text { Wave III } \\
\text { Mean } \pm \text { SD }\end{array}$ & p & $\begin{array}{l}\text { Wave V } \\
\text { Mean } \pm \text { SD }\end{array}$ & p \\
\hline With mask & \multirow[t]{2}{*}{$99 \mathrm{~dB} \mathrm{nHL}$} & $0.12 \pm 0.28$ & \multirow{2}{*}{$0.002^{*}$} & $0.33 \pm 0.25$ & \multirow{2}{*}{0.169} & $0.45 \pm 0.22$ & \multirow{2}{*}{$0.010^{\prime}$} \\
\hline Without mask & & $0.21 \pm 0.25$ & & $0.37 \pm 0.27$ & & $0.75 \pm 0.96$ & \\
\hline With mask & \multirow{2}{*}{$90 \mathrm{~dB} \mathrm{nHL}$} & $0.17 \pm 0.25$ & \multirow{2}{*}{0.608} & $0.34 \pm 0.22$ & \multirow{2}{*}{0.797} & $0.56 \pm 0.17$ & \multirow{2}{*}{$0.046^{*}$} \\
\hline Without mask & & $0.17 \pm 0.19$ & & $0.31 \pm 0.16$ & & $0.49 \pm 0.17$ & \\
\hline With mask & \multirow{2}{*}{$80 \mathrm{~dB} n \mathrm{~nL}$} & $0.22 \pm 0.23$ & \multirow{2}{*}{$0.002^{*}$} & $0.31 \pm 0.19$ & \multirow{2}{*}{0.103} & $0.50 \pm 0.17$ & \multirow{2}{*}{0.001} \\
\hline Without mask & & $0.11 \pm 0.20$ & & $0.25 \pm 0.20$ & & $0.36 \pm 0.17$ & \\
\hline With mask & \multirow{2}{*}{$70 \mathrm{~dB} \mathrm{nHL}$} & $0.19 \pm 0.22$ & \multirow{2}{*}{0.063} & $0.21 \pm 0.18$ & \multirow{2}{*}{0.395} & $0.35 \pm 0.22$ & \multirow{2}{*}{0.331} \\
\hline Without mask & & $0.16 \pm 0.36$ & & $0.25 \pm 0.22$ & & $0.33 \pm 0.19$ & \\
\hline
\end{tabular}


statistically higher (worse) than the AMDT values without mask in all individuals ( $p=0.001$ for high-risk group; $p=0.035$ for lowrisk group). The AMDT values in high-risk group were smaller (better) than the AMDT values in low-risk group $(p<0.001)$.

\section{DISCUSSION}

Noise-related hidden hearing loss is a functional disorder that is seen in individuals who suffer from noise exposure without hearing loss ${ }^{5}$ and is called cochlear synaptopathy. ${ }^{8}$ In a cochlear synaptopathy study on mice, no difference was observed in the hearing thresholds which were measured with auditory brainstem responses, although the half of the synapses between the inner hair cell and spiral ganglion neurons were lost due to noise exposure.22,23 In the synapse loss after noise exposure, while otoacoustic emissions were obtained, the slope of the ABR wave I amplitude was observed at high stimulus levels. ${ }^{1}$

As shown in Figure 3 and Figure 4, we found a decrease in the suprathreshold ABR wave I amplitude with mask in high risk group compared to the low risk group. This decrease with the amplitude value was highlighted in other study about mild and high spontaneous rate fiber loss-induced hidden hearing loss. ${ }^{4}$ In animal studies, it has been reported that the decrease in the wave I amplitude is associated with the synaptic loss with long-term exposure to noise, but the measurement of the wave I amplitude in humans does not provide precise information on the evaluation of synaptopathy. ${ }^{8}$ Studies have indicated that individuals with noise exposure may experience difficulties in terms of supra-threshold processing skills, such as poor understanding and impaired attention. ${ }^{24}$ However, exposure to

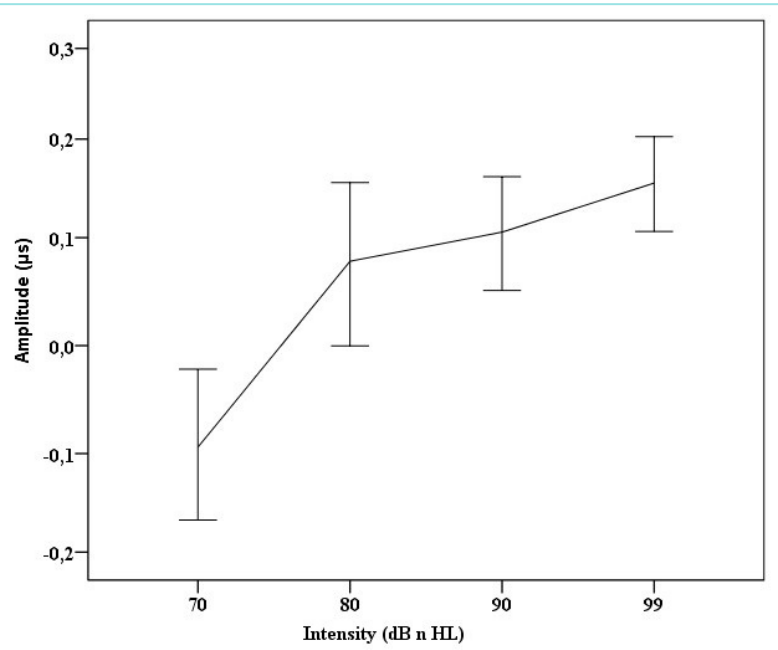

Figure 3. Amplitude findings of wave I of ABR with mask in individuals with high-risk group.

Table 2. Auditory brainstem responses amplitude with/without mask at different stimulus level for the low-risk group

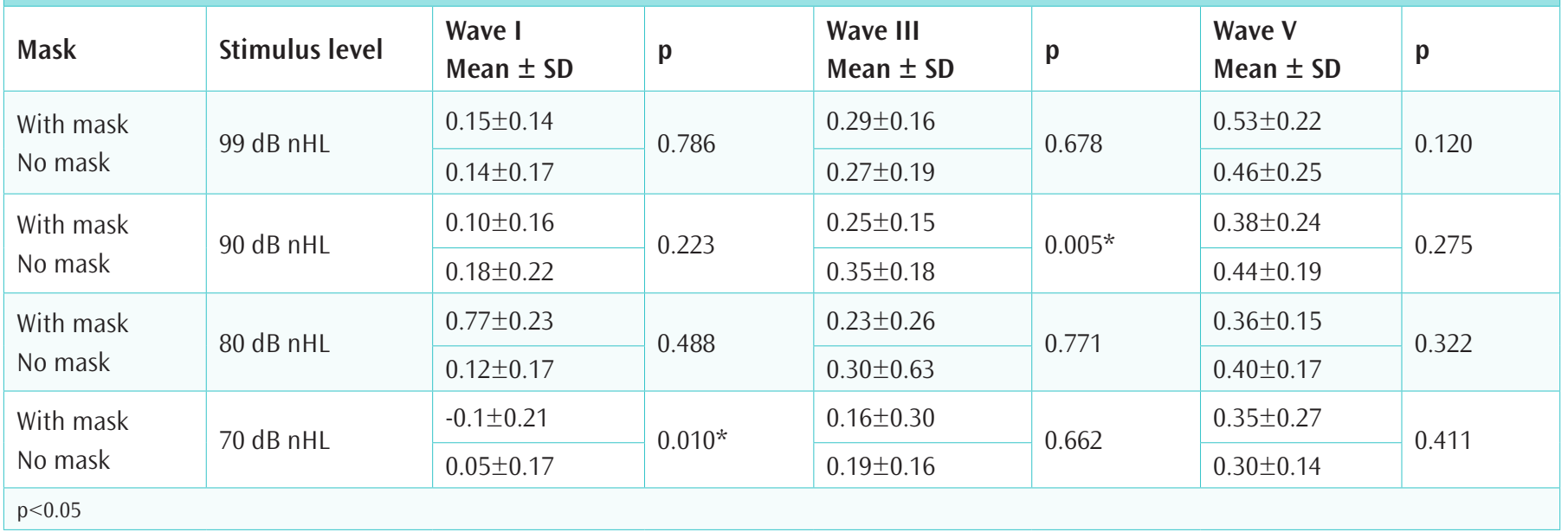

Table 3. Amplitude modulation detection test findings with/without mask for two groups

\begin{tabular}{|l|l|l|l|l|}
\hline \multicolumn{2}{|l|}{ AMDT with mask } & \multicolumn{2}{l|}{ AMDT without mask } \\
\hline Groups & Median (Min, max) & Mean \pm SD & Median (Min, max) & Mean \pm SD \\
\hline High-risk & $-40.1(-46.1,-32.6)$ & $-39.9 \pm 3.16$ & $-40.8(-49.8,-28.8)$ & $-41.2 \pm 4.57$ \\
\hline Low-risk & $-32.2(-41.5,-26.3)$ & $-32.7 \pm 3.58$ & $-37.6(-43.1,-31.1)$ & $-37.5 \pm 3.19$ \\
\hline$p$ & $0.001^{*}$ & & $0.001^{*}$ & $0.001^{*}$ \\
\hline AMDT: Amplitude modulation detection test, min: minimum, max: maximum, $\mathrm{p}<0.05$ & & \\
\hline
\end{tabular}




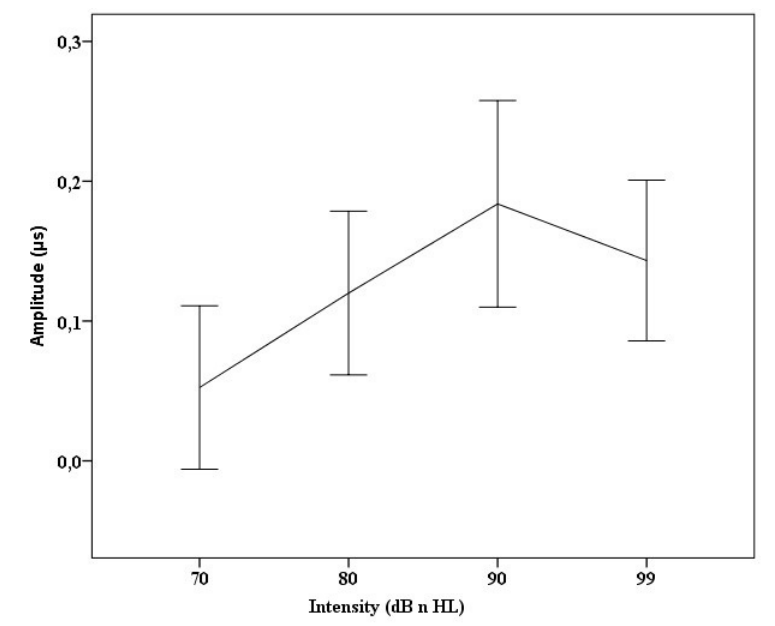

Figure 4. Amplitude findings of wave I of ABR without mask in individuals with high-risk group.

noise did not cause any hearing loss in the high-risk group in our study. We observed the increase in the amplitude of wave I without mask and the decrease with mask as the stimulus level increases $(p<0.05)$. There was a minimum prolongation in the latency of wave $\mathrm{V}$ with mask in comparison without mask in high-risk group was found to be compatible with previous studies. ${ }^{25,26}$ As a finding different from the literature, we found that the masked wave I latency was shorter than the unmasked wave I latency at the suprathreshold levels in the high risk group. In our study, the change in the wave $\mathrm{V}$ latency is accompanied by a decrease in the wave I amplitude in the high-risk group suggested that there may be hidden hearing loss in these individuals. This change mostly reflects the activities of low spontaneous fiber in the presence of background noise. ${ }^{27}$

The amplitude modulation detection threshold was performed in with and without mask and was lower in the high-risk group than those with the low-risk group with mask $(p<0.05)$. This finding suggests that temporal modulation of the sound may impair auditory sensitivity and may be useful in diagnosing hidden hearing loss.

The finding that individuals with the high risk-group with mask had lower AMDT scores than those with the low-risk group may be useful in the diagnosis of hidden hearing loss (Table 3). In studies that were previously conducted, it has been stated that auditory brainstem responses with mask may reflect the efferent auditory system. ${ }^{28}$ In our study, there was no change in the wave I amplitude at stimulus levels of 70 and $80 \mathrm{~dB} \mathrm{nHL}$ with mask for both groups. This finding is consistent with the study conducted by Matas et al. ${ }^{29}$

In our study, individuals with the high-risk group had lower suppression values with contralateral noise than individuals with the low-risk group $(p<0.05)$. This finding is thought that contralateral noise is result in DPOAE suppression by activating efferent neurons as well as a decrease in afferent electric activity. ${ }^{30}$ Marques et al. ${ }^{31}$ highlighted that especially DPOAE data would be beneficial in early diagnosis of cochlear impairments before noise induced hearing loss after the noise exposure.

We thought that a decrease in suppression, I-wave amplitude and AMDT score may lead to hidden hearing loss and thus lead to a decrease in communication skills. The background noise (40dB SPL) which was used in our study can be used to detect low spontaneous fiber loss, especially since it causes suppression of high spontaneous fiber.

\section{CONCLUSION}

Although the ABR test is a gold standard in the diagnosis of occult hearing loss in our study and in the literature, the diagnosis of occult hearing loss with only the ABR test will be insufficient to reveal all the signs of the disease in real terms. Therefore, when diagnosing latent hearing loss, all objective and subjective tests such as amplitude modulation detection tests, otoacoustic emission tests, adaptation test, ABR test should be used together.

Control of noise in high-noise environments such as dental prosthesis laboratories is important for the health of employees due to hearing loss that might be seen in groups with high exposure to noise. In our study, it was observed that ABR wave I amplitude decreased as the intensity increased in the high-risk group. In the low-risk group, it was observed that as the stimulus intensity increased, ABR wave I amplitude wave increased. This is a finding that should be taken into consideration in the diagnosis of occult hearing loss. At the same time, periodically performing audiometric tests of technicians working in these locations and using earplugs to minimize noise exposure of these individuals can help prevent hearing loss. In the process of diagnosing individuals with hidden hearing loss, differentiations in the ABR wave I amplitude, the AMDT and suppression otoacoustic emission (especially DPOAE) may be useful in the early diagnosis of hidden hearing loss.

\section{Main Points}

Noise exposure can cause wave differences in auditory brainstem responses without causing permanent loss of hearing thresholds.

Due to continuous exposure to high intensity noise in dental prosthesis associate degree students can cause permanent loss of auditory nerve cells.

The combined use of Amplitude modulation detection test, otoacoustic emission test, auditory brainstem response and speech in noise tests provides useful information in the diagnosis of occult hearing loss. 


\section{REFERENCES}

1. Mojarad F, MASOUM T, SAMAVAT H. Noise levels in dental offices and laboratories in Hamedan, Iran. 2009.

2. Setcos JC, Mahyuddin A. Noise levels encountered in dental clinical and laboratory practice. International journal of prosthodontics. 1998;11(2).

3. Sampaio Fernandes J, Carvalho A, Gallas M, Vaz P, Matos P. Noise levels in dental schools. European Journal of Dental Education. 2006;10(1):32-7.

4. Kujawa SG, Liberman MC. Synaptopathy in the noise-exposed and aging cochlea: Primary neural degeneration in acquired sensorineural hearing loss. Hearing research. 2015;330:191-9.

5. Borg E, Canlon B, Engström B. Noise-induced hearing loss. Literature review and experiments in rabbits. Morphological and electrophysiological features, exposure parameters and temporal factors, variability and interactions. Scandinavian audiology Supplementum. 1995;40:1.

6. Plack CJ, Léger A, Prendergast G, Kluk K, Guest H, Munro KJ. Toward a diagnostic test for hidden hearing loss. Trends in hearing. 2016;20:2331216516657466.

7. Furman AC, Kujawa SG, Liberman MC. Noise-induced cochlear neuropathy is selective for fibers with low spontaneous rates. Journal of neurophysiology. 2013;110(3):577-86.

8. Kujawa SG, Liberman MC. Adding insult to injury: cochlear nerve degeneration after "temporary" noise-induced hearing loss. Journal of Neuroscience. 2009;29(45):14077-85

9. Kumar UA, Ameenudin S, Sangamanatha A. Temporal and speech processing skills in normal hearing individuals exposed to occupational noise. Noise and Health. 2012;14(58):100.

10. Liberman MC, Epstein MJ, Cleveland SS, Wang H, Maison SF. Toward a differential diagnosis of hidden hearing loss in humans. PloS one. 2016;11(9):e0162726.

11. Schaette R, McAlpine D. Tinnitus with a normal audiogram: physiological evidence for hidden hearing loss and computational model. Journal of Neuroscience. 2011;31(38):13452-7.

12. Morăraşu C, Burlui V, Bortă C, Ignat L, Bortă B, Morăraşu G. The evaluation of sound level in dental practice. Revista medico-chirurgicala a Societatii de Medici si Naturalisti din lasi. 2001;105(4):785-9.

13. Johnson TA, Cooper S, Stamper GC, Chertoff M. Noise exposure questionnaire: A tool for quantifying annual noise exposure. Journal of the American Academy of Audiology. 2017;28(1):14-35.

14. Keskin M. Comparıson Of The Subjects With And Without Nose Sensitıvity, As Determined By The Turkısh Versıon Of Weinstein Noıse Sensitıvity Scale (Wnss) Following Its Adaptatıon Into Turkish. Gazi Unıversıty Instıtute of Health Sciences. 2015.

15. Willeford J. Sentence tests of central auditory dysfunction. Handbook of clinical audiology. 1985;3:404-20.
16. Durankaya SM, Serbetçioglu B, Dalkiliç G, Gürkan S, Kirkim G. Development of a Turkish monosyllabic word recognition test for adults. The Journal of International Advanced Otology. 2014;10(2):172.

17. BECK D, BENITE L. A TWO-MINUTE SPEECH-IN-NOISE TEST Protocol and Pilot Data. Audiology Today. 2019;31(3):28-34.

18. Gorga MP, Neely ST, Dorn PA, Hoover BM. Further efforts to predict puretone thresholds from distortion product otoacoustic emission input/ output functions. The Journal of the Acoustical Society of America. 2003;113(6):3275-84.

19. Ramos JA, Kristensen S, Beck DL. An overview of OAEs and normative data for DPOAEs. Hear Rev. 2013;20(11):30-3.

20. Zilany MS, Bruce IC, Carney LH. Updated parameters and expanded simulation options for a model of the auditory periphery. The Journal of the Acoustical Society of America. 2014;135(1):283-6.

21. Taylor M, Creelman CD. PEST: Efficient estimates on probability functions. The Journal of the Acoustical Society of America. 1967;41(4A):782-7.

22. Hickox AE, Liberman MC. Is noise-induced cochlear neuropathy key to the generation of hyperacusis or tinnitus? Journal of neurophysiology. 2014;111(3):552-64

23. Fernandez KA, Jeffers PW, Lall K, Liberman MC, Kujawa SG. Aging after noise exposure: acceleration of cochlear synaptopathy in "recovered" ears. Journal of Neuroscience. 2015;35(19):7509-20.

24. Brattico E, Kujala T, Tervaniemi M, Alku P, Ambrosi L, Monitillo V. Long-term exposure to occupational noise alters the cortical organization of sound processing. Clinical neurophysiology. 2005;116(1):190-203.

25. Dau T. The importance of cochlear processing for the formation of auditory brainstem and frequency following responses. The Journal of the Acoustical Society of America. 2003;113(2):936-50.

26. Mitchell C, Phillips DS, Trune DR. Variables affecting the auditory brainstem response: audiogram, age, gender and head size. Hearing research. 1989;40(1-2):75-85.

27. Bourien J, Tang Y, Batrel C, Huet A, Lenoir M, Ladrech S, et al. Contribution of auditory nerve fibers to compound action potential of the auditory nerve. Journal of neurophysiology. 2014;112(5):1025-39.

28. Salo SK, Lang AH, Salmivalli AJ, Johansson RK, Peltola MS. Contralateral white noise masking affects auditory N1 and P2 waves differently. Journal of Psychophysiology. 2003;17(4):189-94.

29. Matas CG, Silva F, Leite RA, Samelli AG. Study of suppression effect in the brainstem auditory evoked potential. Pro-fono: revista de atualizacao cientifica. 2010;22(3):281-6.

30. Tomchik SM, Lu Z. Modulation of auditory signal-to-noise ratios by efferent stimulation. Journal of neurophysiology. 2006;95(6):3562-70

31. Marques FP, Costa EAd. Exposure to occupational noise: otoacoustic emissions test alterations. Revista Brasileira de Otorrinolaringologia. 2006;72(3):362-6. 
